\title{
TIEMPO DE HUMANIDADES: NOTAS DE CAMPO (CLIMATICO)
}

\author{
Rainer María Hauser Molina ${ }^{1}$
}

\section{Resumen/Abstract}

En este artículo, trataremos de dar un significado a la complejidad de la era en la que nos encontramos, utilizando como modelo de referencia, el logro más importante de gobernanza policéntrica que la humanidad ha podido concebir en el Antropoceno: la Convención Marco de Naciones Unidas de Cambio Climático (CMNUCC) y sus múltiples instancias orgánicas, para articular ciencia, política y economía. Postulamos que, en su corta existencia, la Convención ha pasado por tres fases principales, cada una de las cuales convoca principalmente, a uno de los diferentes grupos de ciencias en las que tradicionalmente consideramos dividido el conocimiento científico: ciencias básicas, ciencias sociales y ciencias humanas. En este sentido, consideramos en el presente escrito que es una respuesta consistente con el llamado de los tiempos, caracterizado por la búsqueda humanista de significado, expresada a través de las épocas en el concepto de "espíritu", en cuanto definición que rescata los valores de una concepción sistémica, holística y dialógica. La transición a fuentes de Energías Renovables No Convencionales (ERNC) marca un punto de inflexión en el que las humanidades o ciencias humanas, permiten tomar conciencia de la realidad científica del desastre climático causado por el modo de producción capitalista y la magnitud sistémica de su alcance. El establecer propuestas para modificaciones de tal magnitud reverbera en la dimensión espiritual. El carácter universal de las tres plataformas lanzadas en 2015 por la ONU: Riesgo y Desastres de Sendai, Objetivos de Desarrollo Sostenible y Acuerdos de París, así como la introducción más reciente de los Diálogos de Talanoa (COP 23, 2017) -aquí presentados y referidos brevemente-, parecen corroborar el análisis y proyecciones de nuestro artículo.

Palabras clave: cambio climático, UNFCC, IPCC, Humanidades, Diálogos de Talanoa, Acuerdos de París

\section{HUMANITIES TIME: FIELD NOTES (CLIMATIC)}

In this paper, we will try to give a meaning to the complexity of the era in which we are, using as a reference model, the most important achievement of polycentric governance that humanity has been able to conceive in the actual ere of the Anthropocene: the United Nations Framework Convention of Climate Change (UNFCCC) and its manifold organic instances to articulate science, policy and economics. We argue that in the short course of its existence, the Convention has gone through three main phases that differentially convene each one of the three groups of sciences in which we traditionally consider scientific knowledge is divided (basic sciences, social sciences and humanistic sciences).In this sense, we consider in our writing that this is in itself a response consistent with the call of the times, characterized by an humanistic search for meaning, expressed through the epochs in the concept of "spirit", in which it rescues the values of a systemic, holistic, and dialogical conception. The transition to unconventional renewable energy sources marks a turning point in which the humanities or sciences of meaning, allow humanity to become aware of the scientific reality of the climatic disaster caused by the capitalist mode of production and the systemic magnitude of its scope. Establishing proposals for so deep modifications

\footnotetext{
${ }^{1}$ Chileno. E-mail: hausermonju@gmail.com
} 
reverberates on the spiritual scale. The universal character of the three platforms launched in 2015 by the UN: Risk and Disasters of Sendai, Sustainable Development Goals, and Paris Agreements, as well as the more recent launching of the Talanoa Dialogues (COP 23, 2017), here briefly presented and referred, seems to corroborate the analyses and projections of our lecture.

\section{Keywords: climate change, UNFCC, IPCC, humanities, Talanoa Dialogues, Paris Agreements}

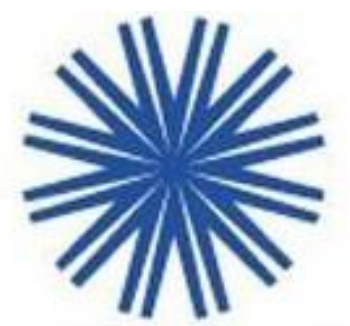

En este escrito trataremos de otorgar un significado ${ }^{2}$, a la complejidad de la era en que nos encontramos, utilizando como modelo referencial, el más importante de los logros de "gobernanza policéntrica" (Ostrom, 2009), que ha sido capaz de concebir la humanidad en el Antropoceno, entendiendo por ello la Convención Marco de Naciones Unidas de Cambio Climático (CMNUCC) ${ }^{3}$ y sus mecanismos de articulación de ciencia, política, y economía (C. Huhnes, Ministro de Energía y cambio Climático, UK, 2008-2012), postulando que en el curso de su existencia, desde 1992, la Convención ha pasado por tres fases principales: Mitigación (1997- 2009), Adaptación (2009-2015) y Acuerdo de Paris (2020-2030), y que los distintos grupos de ciencias (exactas, sociales, humanas) han sido convocadas, cada vez de manera diferente, constatando ahora, con el importante período que marca la transición de las fuentes energéticas a energías renovables, un llamado a las Ciencias del Espíritu (Geisteswissenshaften. o Ciencias Humanas), que marcan el punto de inflexión en que la humanidad toma conciencia de la realidad del desastre climático provocado por el Modo de Producción Capitalista y de la magnitud sistémica de sus alcances.

\section{El punto de ataque. (O desde donde miramos)}

Sin encerrarnos de partida en la caja disciplinaria que tratamos de abrir, estas letras habrían de llevar la impronta de las ciencias humanas, en su irrenunciable búsqueda de significado. "Se dirá, pues, que hay "ciencia humana" no por todas aquellas partes en que se trata del hombre, sino siempre que se analiza, en la dimensión propia de lo inconsciente, las normas, las reglas, los conjuntos significativos que develan a la conciencia las condiciones de sus formas y de sus contenidos.” (Foucault 1966: 355). Hace ya tiempo que son muchos los autores que advierten lo que está ocurriendo en el espacio de nuestros pensamientos

\footnotetext{
2 "Significar es traducir de un código de referencias a otro". (Levi-Strauss 2012).

${ }^{3}$ www.unfccc.int
} 
e interpretaciones y no habría como estar de acuerdo...: "esta divergencia de puntos de vista es la cesura más neta dentro de la tradición intelectual occidental. Por un lado, el pensamiento occidental ha dado nacimiento a la ciencia y, por consiguiente, al determinismo; por otro lado, este mismo pensamiento ha aportado el humanismo, que nos remite, más bien, hacia las ideas de responsabilidad y creatividad". (Prigogine, 2012: )

Advertidos por la Mediología (Debray, 2000) del error común de nuestras epistemologías cartesianas, que enseñan que las ciencias se definen por su objeto, cuando en realidad lo hacen por la mirada que sobre él portamos, buscamos con ellas el diálogo. Así, un árbol será visto como un ente vivo por la biología, como parte de un ecosistema por la ecología, como un conjunto particular de átomos para un químico, como forma de hacer negocios para la economía... Nuestra mirada, querría merecer la pertenencia a una Antropología que fiel a su etimología, se empinara sobre las diferencias, todas demasiado cercanas y llenas de intereses, para expresar los puntos de convergencia y unidad de los saberes y ciencias humanas, en "una Astronomía de las Ciencias Sociales" (Levi-Strauss: 1958: 415).

Así, buscamos "encontrar significado a configuraciones muy diferentes, tanto por el orden de magnitud que por su alejamiento, de aquellas que están en proximidad del observador", para, poniendo un poco de perspectiva, y alejándonos de este maremágnum, invitarlos a mirar la realidad de la realidad a la que refiere este texto, que busca sentido en su narrativa ("memoria, mito, trasmisión de la palabra y del ejemplo, vehículo de la tradición, conciencia crítica del presente, desciframiento del destino de la humanidad, anticipación del futuro o promesa de un retomo...)”, (Foucault, 1966) y que probablemente, más por el cariño que puedan profesarle al leerlo, que por sus virtudes propias, en algunos momentos lo logre.

\section{Sin Poesía}

El Cambio Climático, es el más trascendental de los fenómenos producidos por el hombre. La comprensión de su evolución y el incierto futuro, planteamos, está reflejada en las tres fases por las que ha pasado el tratamiento del tema en la Convención Marco de Naciones Unidas de Cambio Climático (CMNUCC) y el desarrollo de las distintas estrategias científicas, políticas y económicas generadas para abordar las dimensiones de los problemas que plantea. 
Desde luego, la amplitud y el peso específico de los temas abordados, limitan nuestro texto a poco más que una introducción general. Y es que no hay dimensión para describir la magnitud de los fenómenos que nos amenazan. Bien advertidos de la recurrencia apocalíptica y al camino de la profecía, en que se puede transformar un discurso con estos ropajes, haremos lo posible por ajustar la letra a la calma, y así encontremos en el presente una sucesión de momentos anteriores que explican el que hayamos llegado a él. No habrá en nuestro propósito (de "estudiar la anatomía del hombre para entender la anatomía del mono", como decía Karl Marx) ninguna sombra de estar sometidos a la manipulación inevitable de fuerzas deterministas, entonces del destino. No menos cierto, es que en el fárrago inevitable de números, datos y valores con que tratamos de dar validez a nuestros análisis, hemos perdido al humano que siente y entonces, al relato que lo conmueve, lo hace tomar posiciones, lo involucra, lo hace pensar.

\section{La indiscutible evidencia científica}

Probablemente, el informe que entrega cada cuatro años el International Panel of Cimate Change (IPCC) sea la obra científica más importante jamás realizada por la humanidad. En términos ontológicos, porque aborda el mayor desafío que nunca ha enfrentado la vida en el planeta. Epistemológicamente, porque remite al esfuerzo colectivo y continuo en el tiempo de más de 2500 científicos de todos los países, divididos en tres áreas interdisciplinarias que realizan mediciones y actualizan proyecciones y modelamientos sobre los principales ámbitos de impacto del aumento paulatino de temperatura, además se entrega una "síntesis para tomar decisiones políticas". Metodológicamente, por la incorporación de tecnología satelital y de punta en todos los niveles, articulando ciencias exactas y sociales en criterios que permitan traducir sus avances al lenguaje de la política pública como objetivo.

Frente a la evidencia creciente de aumentos de la temperatura y alteraciones de los patrones hidrológicos, así como de otros fenómenos perceptibles en distintos lugares del mundo, la Organización Meteorológica Mundial (OMM) y el Programa de las Naciones Unidas para el Medio Ambiente (PNUMA) constituyeron en 1988 el Grupo Intergubernamental de Expertos sobre el Cambio Climático (IPCC, por sus siglas en inglés). Los principales productos del IPCC consisten en Informes de Evaluación, Informes Especiales, Guías Metodológicas y Documentos Técnicos. Cada informe del IPCC va acompañado de un Resumen para Responsables de Políticas, que se publica en todos los idiomas oficiales de las Naciones Unidas. Tales resúmenes reflejan los conocimientos más recientes en la materia y son redactados de manera comprensible para los no especialistas. 
Los Informes de Evaluación (Assessment Reports: “AR”) constan de varios volúmenes, y proporcionan todo tipo de información científica, técnica y socioeconómica sobre el cambio climático, sus causas, sus posibles efectos, y las medidas de respuesta correspondientes que el estado del conocimiento científico permite. A su vez, las observaciones del sistema climático están basadas en mensuraciones directas y data obtenida de sensores remotos, como satélites y otras plataformas. Observaciones precisas con instrumentos a escala global comenzaron a hacerse desde mediados del siglo XIX para la temperatura y otras variables, incorporando instrumental más comprensivo, diverso y preciso a partir de 1950.

Las reconstrucciones sobre el paleoclima se extienden sobre millones de años y permiten una visión comprensiva de la variabilidad y cambios de "largo aliento" en la atmósfera, el océano, la criósfera y la superficie de la tierra. La comprensión de los recientes cambios en el sistema climático es el resultado de observaciones combinadas, estudios de procesos de retroalimentación y simulaciones de modelo. Se puede decir que con respecto al AR4 (2007), el último AR5 (2013/2014) tiene mejoras sustantivas respecto al detalle y relaciones entre los modelos y componentes del sistema climático, así como respecto a la contribución humana a sus cambios. Resultado: la influencia humana en el calentamiento observado es clara e inequívoca, proviene de las emisiones de Gases con Efecto Invernadero (GEI) y su creciente concentración en la atmósfera.

De esta manera, el AR5 en su Informe de Síntesis del Grupo 1, Bases Físicas del Sistema Climático (2014) establece, en definitiva: "El calentamiento del sistema climático es inequívoco y desde el 1950 muchos de los cambios observados no tienen precedentes en milenios. La atmósfera y el océano se han calentado, las cantidades de nieve y hielo han disminuido, los niveles del mar han subido y las concentraciones de Gases Efecto Invernadero han aumentado".

No se ahondará aquí sobre los detalles del estudio, advirtiendo que las conclusiones son globales y que los trabajos de down-scaling que permitan lograr las precisiones regionales necesarias, se realizan a un ritmo siempre menor que sus efectos, limitándonos por el momento a repetir con sus autores que, de aquí en más, se tiene absoluta certeza científica, sobre el estado y la evolución de cuatro componentes esenciales del sistema climático: 
1) Atmósfera: es seguro que cada una de las últimas tres décadas ha sido sucesivamente más caliente que cualquier década precedente, desde 1850. Es casi seguro (médium confidence) que en el hemisferio norte el periodo entre 1938 y 2012 fue el de los 30 años más cálidos en los últimos 1400 años.

2) Calentamiento del Océano: hay certeza de que el aumento de la temperatura de los océanos domina el aumento de energía que guarda el sistema climático, dando cuenta de más del $90 \%$ de la energía acumulada entre 1971 y 2010 (High confidence). Es también virtualmente seguro que el océano superior (0-700 m) se ha calentado en proporción entre 1971 - 2010 y que posiblemente lo hizo entre 1870-1971.

3) Criósfera: se tiene certeza que, en las últimas dos décadas, las capas de hielo de Groenlandia y la Antártica han ido perdiendo su masa en aumento, que los glaciares se han reducido en casi todos los lugares del mundo y que la cubierta de nieve primaveral sobre la calota polar Ártica y en el hemisferio norte ha decrecido en extensión, también de manera continua.

4) Aumento del nivel del mar: se tiene igualmente certeza que el nivel del mar ha subido más desde mediados del siglo XIX que durante los últimos 2.000 años y que esta tendencia se acrecienta en el tiempo. En los últimos cien años (1901-2010) el nivel del mar ha subido, globalmente, en $0.19 \mathrm{~m}$.

\section{Suma y sigue (pero el planeta tiene sus límites)}

Los signos y señales del Cambio Climático son cada vez más perceptibles en los aumentos de temperatura, cambios en los patrones de precipitación, mayor frecuencia y persistencia de sequías, derretimiento de hielos marinos y glaciares, subida de las cotas de nieve, presencia de fuertes, vientos huracanados, incendios forestales, así como condiciones más impredecibles del clima. Estos cambios están siendo impuestos sobre sistemas ecológicos y sociales ya debilitados por trastornos económicos, crecimiento demográfico y poblacional, malos manejos y prácticas ineficientes en el uso y gestión de la tierra, así como débiles estructuras de gobierno.

La presión adicional ejercida por el cambio climático sobre estos sistemas vulnerables, está poniendo en peligro de manera creciente, las vidas y formas de producir el sustento de personas y sociedades tanto en los países desarrollados como en los que no lo son, produciendo niveles mayores de riesgo global, considerando el potencial de exacerbar las tensiones sociales existentes y contribuir a los conflictos violentos. El cambio climático produce cambios en la percepción general de las cosas, abarcando todos los niveles de nuestra vida social. Esto y sus inevitables consecuencias, políticas y humanas es lo que llamamos Campo Climático. 
El clima del mundo está cambiando y continuará haciéndolo a una velocidad sin precedentes en la historia reciente de la humanidad. Los impactos y riesgos asociados con estos cambios, son reales y presentes: ya están ocurriendo en muchos sistemas esenciales para la vida humana, incluyendo los recursos hídricos, la seguridad alimentaria, zonas costeras y la salud. Aunque las situaciones de desastre, son globales y afectan a todos los países, los países en desarrollo, especialmente aquellos menos desarrollados y las comunidades pobres en general, son las más vulnerables. En los países y comunidades vulnerables, los impactos del cambio climático, plantean una amenaza directa a las personas y su simple supervivencia. No obstante, los efectos devastadores de los eventos extremos, los aumentos de la temperatura y el aumento del nivel del mar, tendrán consecuencias para todos nosotros.

La concentración de energía en la atmósfera y la emisión de Gases Efecto Invernadero (GEI), implica desastres, cuyos costos -que pagamos-, serán exponencialmente mayores con el correr del tiempo. Paradojalmente -como otras veces antaño-, el triunfo de la ciencia viene acompañado del fracaso de la política: la evidencia científica es absoluta, pero el Protocolo de Kioto ha caído en las incertidumbres de un post-Kioto y la comunidad internacional navega en aguas a la vez tímidas y agitadas hacia las decisiones que se generan alrededor del nuevo acuerdo del año 2015, el Acuerdo de Paris, ratificado por 195 países, y del que recientemente, se ha retirado Estados Unidos, en un anuncio que de muchas maneras lo hace tambalear. Este acuerdo de París entraría en vigencia el año 2020.

Frente a la evidencia de la data científica, así como a las campañas de desprestigio de los mismos y la poca resonancia que alcanzaban en la sociedad mundial, la Sociedad Científica Americana, a la que suscribieron distintas asociaciones científicas comprendiendo más del 90\% de los científicos del mundo, publicaron ya en 2010, una carta que pretendía alertar contra el uso malicioso de la información y la relativa ignorancia con que se manejan al respecto las políticas públicas: en general. Los principios aquí establecidos, así como sus conclusiones forman un patrón de certeza que no se ha visto sino reafirmado con los años. Entregamos algunos extractos de ella.

"Estamos profundamente preocupados por la creciente escalada de las agresiones políticas contra los científicos en general y sobre los científicos del clima en particular. Todos los ciudadanos deben comprender algunos hechos científicos básicos. Siempre hay cierta incertidumbre asociada con 
conclusiones científicas; La ciencia nunca prueba absolutamente nada. Cuando alguien dice que la sociedad debe esperar hasta que los científicos estén absolutamente seguros antes de tomar cualquier acción, es lo mismo que decir que la sociedad nunca debería tomar acción. Para un problema tan potencialmente catastrófico como el cambio climático, no tomar ninguna acción representa un riesgo peligroso para nuestro planeta.

Las conclusiones científicas derivan de una comprensión de las leyes básicas apoyadas por experimentos de laboratorio, observaciones de la naturaleza, y el modelado matemático e informático. Al igual que todos los seres humanos, los científicos cometen errores, pero el proceso científico está diseñado para encontrarlos y corregirlos. Este proceso es intrínsecamente contradictorio: los científicos construyen reputación y obtienen reconocimiento no sólo por apoyar la sabiduría convencional, sino aún más por demostrar que el consenso científico está equivocado y que hay una mejor explicación. Eso es lo que hicieron Galileo, Pasteur, Darwin y Einstein. Pero cuando algunas conclusiones han sido profundamente y profundamente probadas, cuestionadas y examinadas, ganan el estatus de "teorías bien establecidas" y a menudo son mencionadas como "hechos".

1. El planeta se está calentando debido al aumento de las concentraciones de gases que atrapan el calor en nuestra atmósfera. Un invierno nevado en Washington no altera este hecho.

2. La mayor parte del aumento de la concentración de estos gases durante el último siglo se debe a las actividades humanas, en especial la quema de combustibles fósiles y la deforestación.

3. Las causas naturales siempre juegan un papel en el cambio del clima de la Tierra, pero ahora están siendo abrumados por los cambios inducidos por el ser humano.

4. El calentamiento del planeta hará que muchos otros patrones climáticos cambien a velocidades sin precedentes en los tiempos modernos, incluyendo las crecientes tasas de aumento del nivel del mar y alteraciones en el ciclo hidrológico. Las crecientes concentraciones de dióxido de carbono están haciendo los océanos más ácidos.

5. La combinación de estos complejos cambios climáticos amenaza a las comunidades y ciudades costeras, a nuestros alimentos y fuentes de agua, ecosistemas marinos y de agua dulce, bosques, entornos de alta montaña y mucho más.” ( http://science.sciencemag.org/content/328/5980/826) 
¿Y entonces, a que se debe la ignorancia?

La dictadura perfecta sería una dictadura que tendría la apariencia de democracia, una prisión sin muros cuyos prisioneros no pensarían en escapar. Un sistema de esclavitud donde, a través del consumo y el entretenimiento, los esclavos tendrían 'amor de su servidumbre'.

Aldous Huxley. El mejor de los mundos (1932)

Pocas dudas podría haber que habiendo alcanzado el conocimiento pragmático y cosificado, económicamente orientado, niveles de control y objetivación tecnológico que resulta difícil imaginar, aun cuando somos testigos, generadores, e indudable parte activa del proceso, el avance irrestricto de una forma histórica de apropiación, ha producido una pérdida, alejamiento, alienación, del ser humano en los tres niveles de su relación con la vida: consigo mismo, con el resto de los humanos y con la naturaleza.

Ese sentido de pérdida, se instala de diversas maneras en una conciencia global -objetivada en la personaque busca y encuentra desesperadamente, significados en medio del caos, la concentración de la riqueza, el descrédito de las instituciones, el cuestionamiento a la razón democrática y la degradación del medio, en manos de un tan gigantesco como recientemente identificado denominador común de todo: el Cambio Climático (Klein, 2014).

A la imagen de las tres etapas históricas y operativas de su concepción y desarrollo orgánico, Mitigación, Adaptación y Energías Renovables (Acuerdo de Paris, COP21, 2015), cada una de estas etapasidentificadas aquí solo con el propósito de establecer una tipología relacional-, responde a la expresión y convocatoria de uno de los tres grupos de ciencias en que se puede clasificar el conocimiento: ciencias exactas, ciencias sociales y ciencias humanas o del significado. Sin ser éste un estudio de epistemología, no puede la teoría prescindir de ella. La tendencia hacia el bienestar, la sustentabilidad de los sistemas vivos, el respeto, en una palabra, la ética, es una preocupación creciente, que trasciende los espacios organizativos para radicar, finalmente, en las personas que los conforma.

En la Convención Marco de Naciones Unidas sobre Cambio Climático (CMNUCC) se ha producido históricamente un tratamiento diferenciado entre las áreas de Mitigación y Adaptación, a las cuales se agrega el Acuerdo de Paris (2015) que agrega como una línea de síntesis, la búsqueda activa de fuentes y 
desarrollos de Energías Renovables No Convencionales (ERNC) y que a éste cambio y cada uno de los anteriores, corresponden también enfoques disciplinarios hegemónicos diferenciados (Ciencias Exactas, Ciencias Sociales, Ciencias Humanas) de los cuales hemos de hacernos parte institucional activa.

Que el Acuerdo de Paris (APA21, diciembre 2015) no sólo corresponde a una transformación definitiva en la percepción de la comunidad internacional con respecto a la realidad absoluta y ya presente del Cambio Climático (AR5, 2014), sino que implica la apertura de un enorme campo de negocios, que más que antes, lleva asociado un ingente acento sobre la apertura de líneas de Investigación y Desarrollo, para participar de la cual se requiere transparencia e innovación.

A estas hipótesis de trabajo, se agrega una certeza que, aunque debiere dar pie a una investigación específica, no podemos dejar de plantear dentro de los elementos claves de una definición de contexto y es que las evidencias de los efectos del Cambio Climático, las pérdidas de vidas y bienes, así como sus costos eventualmente asociados, constituyen una realidad insoslayable, que debe incorporarse de manera urgente al diseño de nuestras herramientas efectivas de política pública.

Las tres fases de la Convención Marco de Naciones Unidas de Cambio Climático (CMNUCC) y sus registros epistemológicos.

\section{Mitigación y Ciencias Exactas}

Una primera fase, desde la creación de la CMNUCC en 1992, estaría conformada por los esfuerzos iniciales por determinar si efectivamente y de qué manera, los Gases Efecto invernadero (GEI) incidían en el calentamiento global y el difícil establecimiento de mecanismos idóneos para combatirlo, cuestión que se vio objetivada por la creación del acuerdo llamado Protocolo de Kioto, llamado así por la ciudad de Japón donde se llevó a cabo la "Conference Of the Parties" (COP) en 1997. El acuerdo, entraría en vigor en 2005, para mantener las emisiones de los seis Gases Efecto Invernadero (GEI). De los cuales, el más conocido y que se ha convertido en una medida "equivalente", es el Dióxido de Carbono o CO2.

Digamos que el Protocolo de Kioto, que ha sido la única instancia “vinculante”, es decir de carácter legal y obligatoria para los países, aunque movilizó enormes esfuerzos de 195 países, y entró en vigor como previsto, nunca contó con la ratificación del Congreso de los USA, quien hasta el 2010 era el mayor emisor de GEI del planeta, siendo ese año superado por China y de esa manera nunca cumplió con sus objetivos de Mitigación. Ello no impidió que algunos países como Alemania; Dinamarca y otros de la comunidad 
europea (EU) si redujesen sus emisiones y sentaran bases sólidas en producción limpia e investigación aplicada en ERNC, que ya conforman el corazón de sus fuentes energéticas.

Dos cuestiones quiero señalar aún sobre la Mitigación. La primera, es que -más allá de mecanismos de traspaso, como el mercado de Bonos de Carbono, (del que como país somos parte activa), y que en estricto rigor esconde responsabilidades tras la posesión de dinero- compromete sólo a los países emisores, es decir industrializados, es decir desarrollados, es decir ricos. De esta manera, recientemente en la conferencia de Bangkok, se ha visto una importante reacción de las organizaciones en la denuncia de estos mecanismos de privatización de los Bienes Comunes. ${ }^{4}$ Lo segundo, es que las instancias de medición y comprobación, puestas en marcha por la convención, apelaron fundamentalmente, a las ciencias exactas, más bien reconocidas como ciencias básicas. Era necesario tener pruebas empíricas y precisas de cuáles eran los efectos de la quema de determinados hidrocarburos fósiles a gran escala, cómo, cuanto y dónde se emitía, para tomar medidas que resultaran apropiadas en consecuencia. Esa fase se terminaría. en la COP15, el 2009.

\section{Adaptación y ciencias sociales}

En efecto, la COP15, (Copenhague, Dinamarca, 2009), pese a la reciente elección de Obama como presidente de los USA y las esperanzas que el mundo se hacía, no lo ratificó el Protocolo de Kioto -el Congreso de los USA rechazó hacerlo-, y Japón anunció su retiro del mismo, seguido luego por Canadá y Australia. Pero junto a ello, y es lo que rescatamos, el AR4 del IPCC de 2007, entregaba datos que implicaban una certeza del 67\% de los efectos del calentamiento global en el planeta y en muchos casos (como el derretimiento de las calotas polares y el proceso de acidificación de los mares), resultados mucho más rápidos que los proyectados ${ }^{5}$. Este cúmulo de situaciones, llevó a que la COP15, viera la creación del Green Fund de Copenhague, que enriquecido en los COP siguientes de Cancún y Durban los años venideros, inició la etapa de Adaptación al Cambio Climático.

\footnotetext{
${ }^{4}$ http://enb.iisd.org/climate/sb48-2/?utm_medium=email\&utm_campaign=2018-09-12\%20-\%20SB42\%20BKK\%20\%20ENB\%20-\%20English\%20-\%20Summary\%20SW\&utm_content=2018-09-12\%20-\%20SB42\%20BKK\%20-\%20ENB\%20\%20English\%20\%20Summary\%20SW+CID_43d3f5f45bfe4049b69d8a5b0f409ba6\&utm_source=cm\&utm_term=httpenbiisdorgclimatesb482 ${ }^{5}$ http://www.ipcc.ch/report/ar4/
} 
La Adaptación supone el reconocimiento de que, cualquier cosa que se pueda hacer, los efectos del Cambio Climático se harán sentir cada vez más y que los países han de tomar medidas y generar Planes Nacionales de Adaptación. Esta segunda fase, marcada por la Adaptación, está dirigida por la atención preferente a los países en vías de desarrollo, que son los más vulnerables a los efectos devastadores de los desastres que conocemos. La Adaptación, convoca de manera preferente a las Ciencias Sociales, quienes, a través de la búsqueda e implementación de metodologías participativas, deben convocar a personas e instituciones, no sólo al diseño y publicación de los planes (como ha ocurrido principalmente entre nosotros), sino a la realización efectiva de las acciones de prevención que implica.

Energías Renovables No Convencionales (ERNC) y Ciencias del Significado.

Marcado por el miedo y la guerra, los atentados que de solo unos días precedieron en Paris a la COP21, así como al "estado de excepción” que a partir de ellos se implementó (y que por cierto continúa hasta hoy...), implicó que 200 mil soldados se tomaran las calles, haciendo que la participación activa de la sociedad civil que se esperaba y se había preparado desde hace tiempo por diversas organizaciones (recordemos la marcha global por el Cambio Climático que se llevó a cabo en las principales ciudades del globo y que en Nueva York convocó a medio millón de personas, repetida este año, en todo el mundo el 8/9/18 ${ }^{6}$ no iba a tener ninguna posibilidad de apoyar el planteamiento de los países más pobres y vulnerables que querían institucionalizar, como parte autónoma de la COP la dimensión Daños y Pérdidas y plantear la exigencia de pago de una Deuda Histórica, a los países ricos, así como impedir el acceso a las Corporaciones Multinacionales, de siempre destacada participación en estos eventos.

Dos millones de personas se esperaban en la COP 21 de Paris, reconocida como la última posibilidad de hacer algo que permitiera frenar el proceso irreversible del Cambio Climático. Gracias a los "lamentables sucesos" que consignamos, se logró un muy aplaudido y resonante acuerdo de transición hacia Sociedades de bajas emisiones de Carbón, sobre la base de compromisos no vinculantes y voluntarios de los países, para no superar $\operatorname{los} 2^{\circ} \mathrm{C}$ de aumento al 2030 y "hacer esfuerzos por mantener la temperatura bajo los $1,5^{\circ} \mathrm{C}^{\prime \prime}$.

\footnotetext{
${ }^{6}$ https://riseforclimate.org/

7 https://unfccc.int/process-and-meetings/the-paris-agreement/the-paris-agreement
} 
Brevemente, digamos que si uno agrega las Intended National Contributions (INDC), que en el marco del Acuerdo de París han sido establecidas y presentadas por los países, se llega a emisiones que superan los $3^{\circ} \mathrm{C}$. Los Estados Unidos de Norteamérica, los USA, se han retirado del Acuerdo y han puesto en duda su continuidad en UN. Ello produjo un remezón interno y la unión de los otros 192 países que cierran filas sobre la necesidad de actuar frente al Cambio Climático acordado. Ello es un elemento diferencial nuevo, que impacta en el rediseño de las fuerzas internacionales, los negocios y las guerras.

El invitado de piedra: la emergencia del Espíritu

Así postulamos que en la Convención Marco de Naciones Unidas sobre Cambio Climático (CMNUCC) se ha producido históricamente un tratamiento diferenciado entre las áreas de Mitigación y Adaptación, a las cuales se agrega el Acuerdo de Paris (2015) que suma como una línea de síntesis, la búsqueda activa de fuentes y desarrollos de Energías Renovables No Convencionales (ERNC) y que a éste cambio y cada uno de los anteriores, corresponden también enfoques disciplinarios hegemónicos diferenciados (Ciencias Exactas, Ciencias Sociales, Ciencias Humanas), de los cuales este texto a su manera da cuenta.

El Acuerdo de Paris (APA21, diciembre 2015) no sólo corresponde a una transformación definitiva en la percepción de la comunidad internacional, con respecto a la realidad absoluta, científicamente comprobad y ya presente del Cambio Climático (AR5, 2014), sino que junto a la apertura de un enorme campo de negocios, que más que antes, lleva asociado un ingente acento sobre la apertura de líneas de Investigación y Desarrollo en Ciencia y Tecnología, con sus consiguientes agregados de innovación y creatividad, vehicula inconscientemente, una transformación que enfrenta al ser humano con la ruptura más radical con el manejo y comprensión de las fuentes de energía que ha utilizado desde que alumbró la caverna, hace medio millón de años.

Así, el Acuerdo de Paris, tiene el carácter de una brújula que indica el norte (lograr economías bajas en CO2 para no sobrepasar el límite de aumento de $2^{\circ} \mathrm{C}$, globales para el 2030), donde cada país, ha trazado su hoja de ruta, de acuerdo con los NDC, que se rigen por el principio de Responsabilidades Comunes pero diferenciadas. Al mismo tiempo, el ya largo, sistemático e ininterrumpido camino de las organizaciones de NU, han desarrollado junto a los compromisos (que es cierto, no han logrado tener carácter vinculante, otro que el de la ética nacional que los sustente), una serie de mecanismos, herramientas y procedimientos, emanados institucionalmente, que permitan enfrentar esta situación ${ }^{8}$. De

\footnotetext{
${ }^{8}$ https://unfccc.int/news/revamped-un-climate-action-portal-to-capture-and-drive-climate-action
} 
esta manera, decimos que a los dos momentos reconocidos de certeza científica del Cambio Climático y el que se requieren políticas públicas consistentes para enfrentarlo, se suma un tercero que debe agregar los mecanismos necesarios para entender por qué, pese a ello, no lo hemos hecho. Se podría decir que la Resiliencia ha hecho emergencia en el sistema.

Hemos señalado como hay una progresión entre la Mitigación y la Adaptación, en los instrumentos de Cambio Climático de UN desarrollados por la Convención y su ratificación por los gobiernos y medidas para implementarlo. Diversas iniciativas gubernamentales se vinculan a esta problemática, siendo la más importante, el Plan Nacional de Adaptación al Cambio Climático (2014) que tiene partes sectoriales más antiguas, como la Adaptación en la Agricultura. En él, instituciones y servicios se relacionan también con tareas específicas. La apertura inminente de líneas de negocios para hacer el cambio de nuestras fuentes generadoras de energía, basadas sobre Hidrocarburos Fósiles (HCF) a Energías Renovables No Convencionales (ERNC) ${ }^{9}$, abrirá la posibilidad de utilizar las enormes capacidades de nuestro país en energía fotovoltaica, geotermia, eólica y mareomotriz que, de no producirse junto a una profunda toma de conciencia de empresarios y comunidades o/y un fuerte y acorde rediseño y fortalecimiento de nuestros sistemas jurídicos de defensa medio ambiental, significará que no aprendimos a pensar de manera diferente y solo dimos un nuevo paso de negocios, vendiendo a grandes precios los salvavidas en el naufragio.

Pero, además, de manera muy significativa, ello se produce en medio de un "clima social" marcado por las amenazas de guerra mundial y crecientes desastres de los cuales, aunque ahora entendemos las causas, no dejan de afectar a nuestras poblaciones. De allí que la Encíclica Papal Laudato Sii, El cuidado de nuestra casa Común (2015), haya venido a hacer explícita en el mundo cristiano, esta preocupación sistémica que ya se había manifestado en la espiritualidad de los Pueblos Originarios, siempre los más afectados por los avances de un "progreso" construido largamente a sus expensas y que en sus tierras ancestrales encontró las fuentes materiales de su acumulación primitiva.

Las Constituciones de Ecuador, Bolivia y Nueva Zelandia, han marcado una pauta de reconocimiento de la dimensión espiritual (del valor trascendente y superior de la naturaleza) que debiéramos seguir y que sigue sus avatares de marcar la pauta de los pasos más avanzados.

${ }^{9}$ www.accionempresas.cl 


\section{Los Diálogos de Talanoa.}

La COP 21 de París (CMNUCC, 2015), será recordada por el gigantesco salto qué significó para los tomadores de decisiones en política el reconocimiento de los datos de la ciencia, en cuanto a los orígenes y consecuencia del cambio climático, por cuánto fue allí acordada la necesidad de realizar una transición energética hacia fuentes de energía renovables no convencionales (ERNC), que permitiera controlar el avance tendencial irresistible de emisiones de gases efecto invernadero (GEI), para qué al 2030 el aumento global promedio de temperatura no supere los 2 grados centígrados.

Así, sobre la base de la evidencia científica (AR5, IPCC), a fines del año 2015 se consignó el Acuerdo de París. Acuerdo voluntario que de alguna manera viene a sustituir el Protocolo de Kioto (2007 -2012). El acuerdo de París fue suscrito por Chile, que se comprometió a reducir sus emisiones en un 35\% en el período que va del 2020 al 2030.

Los gigantescos problemas de coordinación y voluntades, que supone -y supondrá en los años a venir-, el cambio de energías producidas por la extracción de hidrocarburos fósiles (HCF) hacia energías limpias, no podía dejar sin impactar este mundo convulsionado en el cual vivimos, y qué encontró en el retiro de los Estados Unidos del acuerdo, en el 2017, su hecho más relevante. Anotemos, sin embargo, que el retiro de los Estados Unidos no sólo significa que ese país no considera ya en la toma de decisiones, como hemos dicho, los datos irrefutables de la ciencia, sino que, además, se aísla de manera significativa de los espacios dialógicos y de negociación construidos por la comunidad internacional, produciendo un aislamiento externo que sin duda no podrá mantenerse en el tiempo.

Por otra parte, el mismo año, ha visto cómo los países se han puesto de acuerdo para el lanzamiento de otras dos "plataformas universales" que, aunque permiten sustentar una cierta esperanza en el futuro del planeta, no deja de provocar inquietudes por las formas que ha de asumir la coordinación y coherencia en que la gobernanza de instituciones, organizaciones diversas de la sociedad civil, públicas y privadas, empresas y personas, han de ser convocadas al cumplimiento de sus fines. Nos referimos al Marco de Sendai, sobre Prevención de Riesgos y Desastres y a los Objetivos de Desarrollo Sustentable (ODS). 
Recordemos que el objetivo de los Diálogos de Talanoa es compartir historias y relatos en un espíritu constructivo y transparente, para encontrar soluciones en aras de un bien común. De esta manera, implican un objetivo de largo plazo, cual es la construcción común de una historia -hasta ahora hecha imposible por las inequidades de todo tipo- y una metodología de trabajo universal, que puede muy bien ser aplicada a los distintos niveles de nuestras relaciones programáticas.

Respondiendo las tres preguntas orientadoras de "Dónde nos encontramos", “dónde queremos llegar" y “cómo llegamos allí, la tradición cultural del Pacífico y de las Islas Fidji, rescata de una particular manera, que por cierto es común a nuestros pueblos originarios, una compleja y rica problemática que en la historia de las ciencias resumió el sociólogo Egon Guba (Guba, 1998), y hacemos nuestra, señalando que al definir un ámbito de atención, se deben establecer tres enfoques complementarios:

a) la dimensión ontológica, (¿cuál es la naturaleza del objeto que consideramos?);

b) la dimensión epistemológica (¿desde qué perspectiva nos constituimos como sujeto y estamos considerando el objeto?); y

c) la dimensión metodológica (¿cómo se articulan esas dos dimensiones en un modelo de práctica?).

Estudios recientes ponen de relieve la sistematización del trabajo así recogido por las instancias internacionales y su pertinencia en cuanto herramienta para la generación de confianza en las organizaciones. En otro ámbito, directamente relacionado, se ha reconocido recientemente el rol de los relatos, como fundamental en la acción comunitaria, y como material de apoyo para la toma política de decisiones. (Sundin et al. Environmental Evidence, 2018).

De esta manera, Naciones Unidas, pretende crear conciencia y facilitar la acción, a través de la máxima participación ciudadana, en todos los países, de la muy interesante recuperación del relato como forma histórica. Con los Diálogos de Talanoa, y por paradojal que pareciere, se dan los primeros pasos sustantivos de respuesta colectiva, en la implementación concreta de los compromisos nacionales. A través de este ejercicio centrado en las personas, se contempla crear la necesaria conciencia acerca de cambios en los patrones de consumo, que permita la incorporación de sistemas distributivos de energía renovable, a través de modelos cooperativos sostenibles, y otros, que complementen las transiciones energéticas voluntarias de cada país, expresadas en las Contribuciones Nacionales Determinadas (INDC) 2020-2030. 
Referencias bibliográficas

Cleary, T. a. (1977). The Blue Cliff Record (Pi Yen Lu). Boulder and London: Shambhala.

Debray, R. (2000). Introduction a la Mediologie. Paris: Albin Michel.

Foucault, M. (1966). Les mots et les choses. Paris: Gallimard.

Guba, E. (1998). The Paradigm Dialogue. London: Sage.

Klein, N. (2014). This Changes Everything. New York: Barnes and Noble.

Levi-Strauss, C. (1958). Anthropologie Structurale. Paris: Plon.

(1970). Anthropologie Structurelle Deux. Paris: Plon. (2012) Mito y significado, Madrid, Alianza

Ostrom, E. (2009). Beyond market and the states. USA: Indiana University.

Prigogine, I. (2012). El nacimiento del tiempo. Barcelona: Tusquets.

\section{URLs}

www.ipcc.int

http://science.sciencemag.org/content/328/5980/826

https://riseforclimate.org/

https://unfccc.int/process-and-meetings/the-paris-agreement/the-paris-agreement

http://enb.iisd.org/climate/sb48-2/?utm_medium=email\&utm_campaign=2018-09-12\%20-

$\% 20 \mathrm{SB} 42 \% 20 \mathrm{BKK} \% 20-\% 20 \mathrm{ENB} \% 20-\% 20$ English $\% 20-\% 20$ Summary\%20SW\&utm_content=201809-12\%20-\%20SB42\%20BKK\%20-\%20ENB\%20-\%20English\%20-

\%20Summary\%20SW+CID_43d3f5f45bfe4049b69d8a5b0f409ba6\&utm_source=cm\&utm_term=httpen biisdorgclimatesb48-2

http://www.ipcc.ch/report/ar4/

http://www.anthropocene.info/resilience-thinking.php

https://unfccc.int/news/revamped-un-climate-action-portal-to-capture-and-drive-climate-action

http://w2.vatican.va/content/francesco/es/encyclicals/documents/papa-francesco_20150524_enciclicalaudato-si.pdf 


\section{www.portalanoa.blogspot.com}

www.accionempresas.cl

http://www.cl.undp.org/content/chile/es/home/sustainable-development-goals.html https://environmentalevidencejournal.biomedcentral.com/articles/10.1186/s13750-018-0116-4 http://www4.unfecc.int/Submissions/INDC/Submission\%20Pages/Submissions.aspx 